\title{
ADAPTIVE FINITE ELEMENT METHODS FOR TWO-DIMENSIONAL PROBLEMS IN COMPUTATIONAL FRACTURE MECHANICS
}

\author{
J. B. MiN, $\dagger_{+}$J. M. Bass $\S$ and L. W. Spradley $\uparrow$ \\ $\nmid$ NASA/Marshall Space Flight Center, MSFC, AL 35812, U.S.A. \\ §Computational Mechanics Company, Inc., Austin, TX 78752, U.S.A. \\ ๆAdaptive Research Corporation, Huntsville, AL 35805, U.S.A.
}

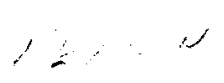

(Received 15 May 1992)

\begin{abstract}
Some recent results obtained using solution-adaptive finite element methods in two-dimensional problems in linear elastic fracture mechanics are presented. The focus is on the basic issue of adaptive finite element methods for validating the new methodology by computing demonstration problems and comparing the stress intensity factors to analytical results.
\end{abstract}

\section{INTRODUCTION}

One of the most difficult analytical challenges in engineering mechanics is the modeling of flawed structures and the computation of the structural response and flaw propagation. These models are generally quite large for many space vehicle structures. The commercially available codes are often used to model the unflawed structure and to perform a stress analysis. These large models must then be reconstructed manually to introduce a flaw and to remesh the problem for accurate modeling of the flaw. One of the most technically challenging areas is fatigue crack propagation in these structural components. Specifically, fatigue life prediction error sources such as the stress intensity factors (SIF) are difficult to quantify. Practically, SIFs are calculated either from handbooks or other simplified equations. However, these equations are applicable only for structural components that approximate the defined numerical and geometric conditions. Furthermore, handbooks do not usually consider mixed mode crack situations which are common for actual structures. Thus, there is a need to minimize the error associated with SIF values and have them integrated into fatigue models for improved fatigue life predictions.

Much of contemporary fracture analysis still follows classical ideas. Modeling of complex geometries has been difficult and conventional computational procedures cannot provide accurate stress predictions for bodies of complex shape. Many fracture theories are developed with only simple stress states in mind and are seldom factored into realistic stress environments of the type actually experienced in working

† To whom all correspondence should be addressed. structural components. In concept, many of the shortcomings of classical fracture analysis may be overcome through the development of more sophisticated computational models. By using new finite element capabilities and new concepts in fracture mechanics, it should be possible to study a variety of basic issues connected with fracture and crack growth. These include the use of more elaborate models of material constitution and component geometry, more general crack initiation and growth criteria, more accurate methods for prediction crack development, and more physically reasonable models of crack arrest mechanisms. Therefore, an effort has been made on the development of the efficient and accurate modeling technique of large space structures containing flaws using solution-adaptive methods. These procedures, using finite element methods, automatically adjust the grid points for refinements of meshes of quadrilateral elements to produce a minimum error solution. A viable approach is to develop a new fracture mechanics analysis tool which is based on modern adaptive finite element methods. The primary goals of this study are to: (1) develop an advanced and reliable numerical method for performing linear elastic analysis of flawed structural components; (2) validate the new methodology by computing demonstration problems and comparing the stress intensity factor to analytical results.

As a result, efficient solution-adaptive algorithms are derived which are suitable for large-scale computations for the solution of confined crack regions and a two-dimensional fracture mechanics analysis code is developed in which a discrete least-squares algorithm and an energy release method are implemented and validated. Demonstration problems are also computed and the SIFs compared with analytical formula. The agreement is reasonably good and thus 
provides validation of the new methodology. A general procedure and methods employed are given in Secs 2 and 3, and the results of demonstration problems are given in Sec. 4.

\section{ADAPTIVE COMPUTATIONAL METHODS}

\subsection{Adaptive schemes}

Adaptive methods, which are numerical schemes which automatically adjust themselves to improve numerical solutions, are generally composed of three key ingredients. The first of these is an adaptive scheme which is capable of dynamically modifying the structure of the computational grid. The second crucial component is an error estimator or error indicator which is used to identify regions of the computational domain which contain relatively large numerical errors. The final necessary ingredient is an adaptive methodology which combines the mesh modification schemes with the error estimators to produce a robust and efficient refinement sequence.

The first work on adaptive finite element methods was presented in 1971 by Oliveira [1] which discussed grid optimization by minimizing the energy through optimal node redistribution. This type of approach, node redistribution, is the basis of moving mesh adaptive methods ( $r$-methods) developed for both solid mechanics problems and flow analysis [1-8]. Following this work, several other adaptive finite element schemes have emerged which include $h$ refinement methods, which subdivide the elements of the computational domain [9-13], $p$-enrichment methods, which increase the polynomial order of the approximations [14-18], and $h-p$ methods, which increase the polynomial order, as well as subdivide the elements comprising the grid [19-23]. In these schemes, the mesh is automatically refined when the local error indicator exceeds a preassigned tolerance. The $h$-scheme presents a difficult data management problem, since they involve a dynamic regeneration of the mesh, renumbering of grid points, cells or elements, and element connectivities as the mesh is refined. Since it has been proven that the $h$-methods can be very effective in producing near-optimal meshes for given error tolerances and also be used to coarsen a mesh (use large mesh cells and thereby reduce the number of unknowns) when the local-error becomes lower than an assigned lower-bound tolerance among the adaptive methods [9], the $h$ method is taken in this study. A sample calculation obtained with our $h$-method is shown in Fig. 1 for a uniformly loaded plate containing a crack. Our procedure dynamically refines the mesh, assigning large elements where the error is small and small elements where the error tends to be large, thus capturing the singularity of the crack tip. Computed contours of the $\sigma_{y}$ stress component are also shown in the figure.
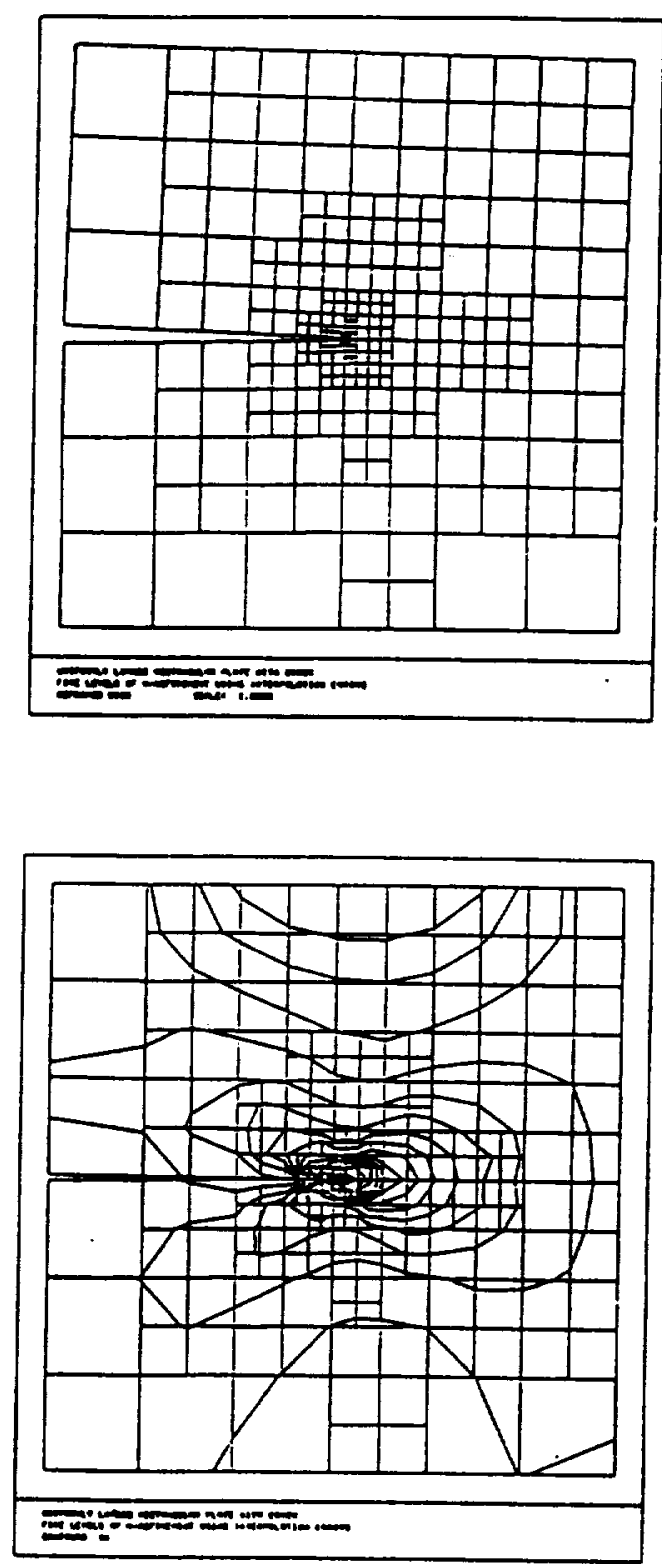

Fig. I. Uniformly loaded crack plates: $h$-adapted mesh and $\sigma_{y}$ stress contours. Note that the model is not exactly symmetric for the top and bottom parts.

\subsection{Error estimation}

An adaptive scheme is essentially useless without a reliable, accurate, and efficient error indicator. The error indicator provides an estimate of the local error in the numerical solution and an indication where the grid should be adapted. In general, there are two distinct types of error estimators, interpolation error estimators and residual error estimators. The interpolation error estimates typically provide only a very crude estimate of the error, but usually provide a very good indication of the relative errors and are quite inexpensive to implement. Residual methods, on the other hand, typically provide a much better estimate of the actual error in the numerical solution but are 
generally much more computationally expensive. Therefore, the interpolation error estimates are taken in this study. Some general properties of the distribution of error in finite element meshes and the use of so-called interpolation error estimates shall be discussed here. This easily implementable local estimator sometimes provides only a rather crude estimate of the actual local error but can be divised to give a correct indication of the relative error between successive meshes or approximation orders and, thus, correctly direct an adaptive strategy to systematically reduce local error. For a more detailed discussion of interpolation theory, see $[24,25]$. The simplified theory of this error estimates follows.

Let $u$ be a smooth function defined over a regular domain $\Omega \subset \Re^{2}$. The $W^{r . p}(\Omega)$-seminorm of $u$ is defined by

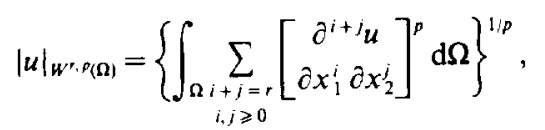

where $1<p<\infty$ and $r$ is a non-negative integer. For the special case of $p=\infty$, which is also of interest, the $W^{r . p}(\Omega)$-seminorm is given by

$$
|u|_{W^{r, x}(\Omega)}=\max _{x \in \Omega} \max _{\substack{i+j=r \\ i, j \geqslant 0}}\left|\frac{\partial^{i+j} u(x)}{\partial x_{1}^{i} \partial x_{2}^{j}}\right| .
$$

With these definitions of the seminorm, the Sobolev norm of $u$ is then

$$
\|u\|_{W^{r, p}(\Omega)}=\left\{\sum_{k=0}^{r}|u|_{W^{k, p(\Omega)}}\right\}^{1 / p} .
$$

Let $G$ be an arbitrary convex subdomain (a finite element) of $\Omega$ over which $u$ is interpolated by a function $\tilde{u}_{h}$ which contains complete piece polynomials of degree $k$. Then it can be shown that the local interpolation error in the $W^{m, p^{\prime}}(\Omega)$-seminorm is

$$
\begin{aligned}
& \left|u-\tilde{u}_{h}\right|_{w^{m, p^{\prime}(G)}} \\
& \quad \leqslant C \cdot \frac{h^{k+1}}{\gamma^{m}} \cdot h^{\left(n ; p^{\prime}\right)-\langle n ; p)} \cdot|u|_{W^{k+1 . p(\Omega)}},
\end{aligned}
$$

where $h$ is the diameter of domain $G, \gamma$ is the diameter of the largest sphere that can be inscribed inside $G$, $n$ is the dimension of the domain $\Omega, C$ is a constant independent of $h$, and $p^{\prime}$ is $p /(p-1)$. If $\gamma$ is proportional to $h$, and if it remains proportional in refinements of $G$ defined by parametrically reducing $h$, we have

$$
\left|E^{h}\right|_{m, p^{\prime}, G} \leqslant C \cdot h^{\left(n ; p^{\prime}\right)-(n ; p)+k+1-m} \cdot|\boldsymbol{u}|_{k+1, p}
$$

with

$$
|\cdot|_{m, p^{\prime}, G}=|\cdot|_{W^{m, p^{\prime}(G)}} \text {, etc. and } E^{h}=u-\tilde{u}_{h} .
$$

Suppose that $u$ on the right-hand side is now replaced by a finite element approximation $u_{h}$ and $\left|u_{h}\right|_{k+1, p}=|u|_{k+1, p}+O(h)$. Then the above estimate indicates that the local error in the $W^{m, p}(G)$-seminorm is proportional to the error indicator,

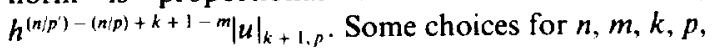
and $p^{\prime}$ of interest are:

$$
\begin{aligned}
& \text { (i) } n=2, m=0, k=1, p=p^{\prime}=2 \text { then } \\
& \left|E^{h}\right|_{L^{2}(G)} \leqslant C \cdot h^{2} \cdot|u|_{2,2, G} .
\end{aligned}
$$

In this case, one must approximate the $W^{2,2}$-seminorm of $u$ over $\Omega$; i.e., the $L^{2}$-norm of the second partial derivatives of $u$. The error indicator $\theta_{e}$ is then set equal to $\left|E^{h}\right|_{L 2\left(\Omega_{e}\right)}$ for finite element $\Omega_{e}$.

(ii) $n=2, p=\infty, p^{\prime}=1, k=0, m=0$ then

$$
\begin{aligned}
\left|E^{h}\right|_{L^{\prime}(G)} & =C \cdot h^{2} \cdot\left|E_{\text {average }}^{h}\right| \leqslant C \cdot h^{3} \cdot|u|_{1, x, G} \\
& \leqslant C \cdot h^{3} \cdot \max _{x \in G}|\nabla \cdot u(x)|
\end{aligned}
$$

then we have for the error indicator $\theta_{e}$

$$
\left|E^{h}\right|_{\text {average over } \Omega_{e}} \approx \theta_{e}=h \cdot \max _{x \in G}|\nabla \cdot u(x)|
$$

In all of these cases, it is also possible to estimate the constant $C$. While we shall not describe how this is done (see [25]), our experience is that it is a worthwhile computation that can lead to schemes with good effectivity indices.

\subsection{Adaptive methodology}

We shall now suppose that an error indicator $\theta_{e}$ can be calculated for each finite element $\Omega_{e}$ in a given mesh. The error indicator is, in general, a real number representing the local error on a suitable norm, and it is computed using one of the procedures described in the next section.

The decision to refine the mesh is based on whether or not local-indicators exceed preassigned tolerances and can be summarized by the following steps.

h-Refinement/unrefinement methodology. The $h$ procedure involves the following steps:

1. For a given domain $\Omega$, a coarse finite element is constructed which contains only a number of elements sufficient to model basic geometric features of the domain.

2. As the adaptive process is designed to handle groups of four elements at a time, a finer starting grid may be generated by a bisection process, if desired, to obtain an initial set of element groups.

3. The numerical solution is calculated on this initial coarse grid, and the error indicators $\theta_{e}$ are computed over all $M$ elements in the grid. Let

$$
\theta_{\max }=\max _{1 \leqslant r \leqslant M} \theta_{\ell}
$$


4. The groups of elements are scanned and the group errors are computed

$$
\theta_{\text {Group }}^{k}=\sum_{k=1}^{p} \theta_{e_{k}},
$$

where $e_{k}$ is an element number in group $k$ and $p=4$.

5 . Error tolerances are defined by two real numbers, $0<\alpha, \beta<1$. If

$$
\theta_{e} \geqslant \beta \cdot \theta_{\max }
$$

element $e$ is refined. This is done by bisecting element $e$ into four new subelements. If

$$
\theta_{\text {Group }}^{k} \leqslant \alpha \cdot \theta_{\max }
$$

group $k$ is unrefined by replacing this group with a single new element with nodes coincident with the corner nodes of the group.

This general process can be followed by any choice of an error indicator. Moreover, it can also be implemented with any boundary frequency in the solution of transient problems or problems with time-dependent boundary conditions.

\section{CALCULATIONS OF SIFs}

Fracture mechanics within the linear theory of elasticity is known as linear elastic fracture mechanics (LEFM). Linear elastic fracture analysis is valid if the assumption of small-scale yielding, namely when the inelastic region in the vicinity of each crack tip is well within the range of validity of the asymptotic solutions of the crack in an infinite elastic medium. Assuming that the crack faces are free of traction, the asymptotic expressions for the neighborhood of the crack tip are given in [34]. In this study the stress intensity factors were calculated using one of the following methods.

\subsection{Discrete least-square fits}

Consider a patch of elements around the crack tip as shown in Fig. 2; for example, we may define

$$
\Omega_{\mathrm{tip}}=\left\{x \in \Omega_{e}|| x_{e}^{\text {cent }}-x^{\mathrm{tip}} \mid \leqslant R(h)\right\} .
$$

Let us define the discrete least squares functional

$$
\begin{aligned}
J\left(K_{\mathrm{l}}, K_{\mathrm{Il}}\right)=\sum_{j=1}^{\text {nintp }}\left(\sigma_{j}^{h}-\right. & \left.\sigma_{j}^{\text {asym }}\right)^{T} \\
& \times D^{-1} \cdot\left(\sigma_{j}^{h}-\sigma_{j}^{\text {asym }}\right) W_{j} .
\end{aligned}
$$

Here, $x_{e}^{\text {ent }}$ is the position vector for the centroid of element $\Omega_{e}, x^{\text {tip }}$ is the position vector for the tip of the crack, $R(h)$ is the radius of the disk which includes the centroids of the element of the subdomain, which is assumed to be a function of the mesh size $h, \sigma_{j}^{h}=\sigma^{h}\left(x_{j}\right)$ denotes the approximate stress vector at point $x_{j}, \quad \sigma_{j}^{\text {asym }}=\sigma^{\text {asym }}\left(x_{j} ; K_{1}, K_{\mathrm{Il}}\right)$

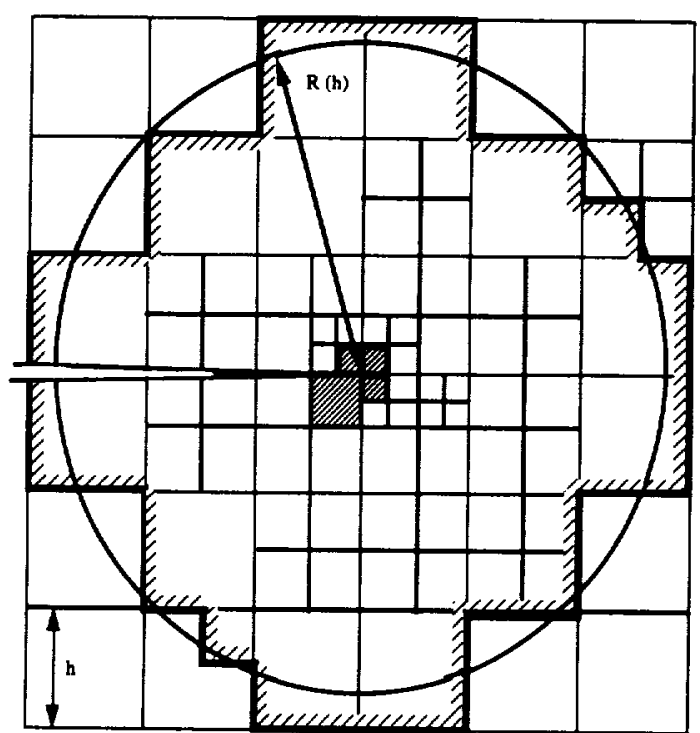

Fig. 2. A subdomain which includes the elements of the grids with centroids in the interior of the disk $\left\{x \in \Omega \| x^{\text {tip }}-x \mid \leqslant R(h)\right\}$. The elements which include the crack tip appear shaded.

denotes the asymptotic stress vector at point $x_{j}, D^{-1}$ is the inverse of the material modulus matrix, $x_{j}$ denotes an investigation point from the set of integration points of the elements which form $\Omega_{\text {ipp }}, W$, denotes the weight for the $j$ th integration point, and nintp denotes the total number of integration points in the subdomain $\Omega_{\text {tip }}$.

The components of the stress $\sigma^{\text {asym }}\left(x, K_{1}, K_{\mathrm{II}}\right)$ depend linearly on the SIFs; their analytical expression is given in [33]. Approximate values for the SIFs are extracted as minimizers of the discrete least squares functional $J\left(K_{\mathrm{l}}, K_{\mathrm{II}}\right)$; the condition of a stationary value leads to a two-by-two system of linear equations for the approximate values of $K_{\mathrm{l}}$ and $K_{\mathrm{ll}}$, namely

$$
\begin{aligned}
\frac{\partial J}{\partial K_{\mathrm{I}}}=-2 \cdot \sum_{j=1}^{\text {nintp }} \frac{\partial\left(\sigma_{j}^{\text {asym }}\right)^{T}}{\partial K_{\mathrm{l}}} \\
\quad \times D^{-1} \cdot\left(\sigma_{j}^{h}-\sigma_{j}^{\text {asym }} \cdot W_{j}\right)=0
\end{aligned}
$$

$$
\begin{aligned}
\frac{\partial J}{\partial K_{\mathrm{II}}}=-2 \cdot \sum_{j=1}^{\text {nintp }} & \frac{\partial\left(\sigma_{j}^{\text {asym }}\right)^{T}}{\partial K_{\mathrm{II}}} \\
& \times D^{-1} \cdot\left(\sigma_{j}^{h}-\sigma_{j}^{\text {asym }} \cdot W_{j}\right)=0 .
\end{aligned}
$$

A bilinear element is employed to compute the approximate finite element solution and the discrete least squares functional in each element is defined using $1 \times 1$ and $2 \times 2$ Gauss integration rules. Let $K_{1}^{1}$, $K_{\mathrm{II}}^{\mathrm{l}}$, and $K_{\mathrm{I}}^{2}$, and $K_{\mathrm{II}}^{2}$, denote the extracted values of the SIFs corresponding to the use of the $1 \times 1$ and $2 \times 2$ rules in the definition of the functional. Since the definition of the $K^{1}$ values involves superconvergent values of stress at the centroids of the elements, 


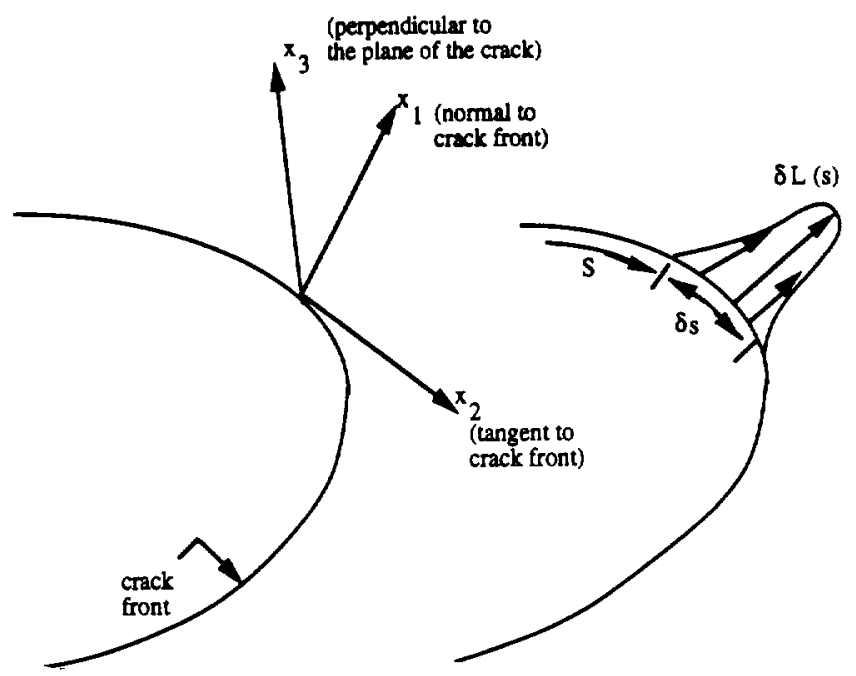

Fig. 3. Planar crack in three dimensions.

the following hypothesis was taken from the numerical experiments to determine that the error in the $K^{2}$ values of the SIFs can be estimated by comparing them with the $K^{1}$ values

$$
K-K^{2} \simeq K^{1}-K^{2} .
$$

\subsection{Equivalent domain integral methods}

Equivalent domain methods were developed by Shih, Moran and co-workers [26-28]. Li et al. [26] derived a volume integral expression for the energy release rate which can be naturally employed to extract the energy release rate along threedimensional crack fronts from three-dimensional finite element calculations. Also, the two-dimensional area integral analog of the volume integral expression was described. The energy release rate is defined as follows: Consider a planar crack front with a continuously turning tangent as shown in Fig. 3. Let $\delta L(s)$ denote the crack advance at the points in the direction normal to the crack front and $\mathrm{d} s$ denote the length of a crack element along the crack front. Then, within first-order terms in $\delta L(s)$ and $G(s)$, namely the pointwise energy release rate at the location $s$ of the crack front, defined by (see [26] and references therein)

$$
\int_{\text {crack front }} G(s) \cdot \delta L(s) \mathrm{d} s=-\delta \pi .
$$

Here $\delta \pi$ denotes the change in the total potential energy due to the local advance of the crack given by $\delta L(s)$. The above definition may be considered as a weak or variational statement satisfied by the energy release rate function, which is defined along the crack front. Li et al. [26] suggested a volume-integral

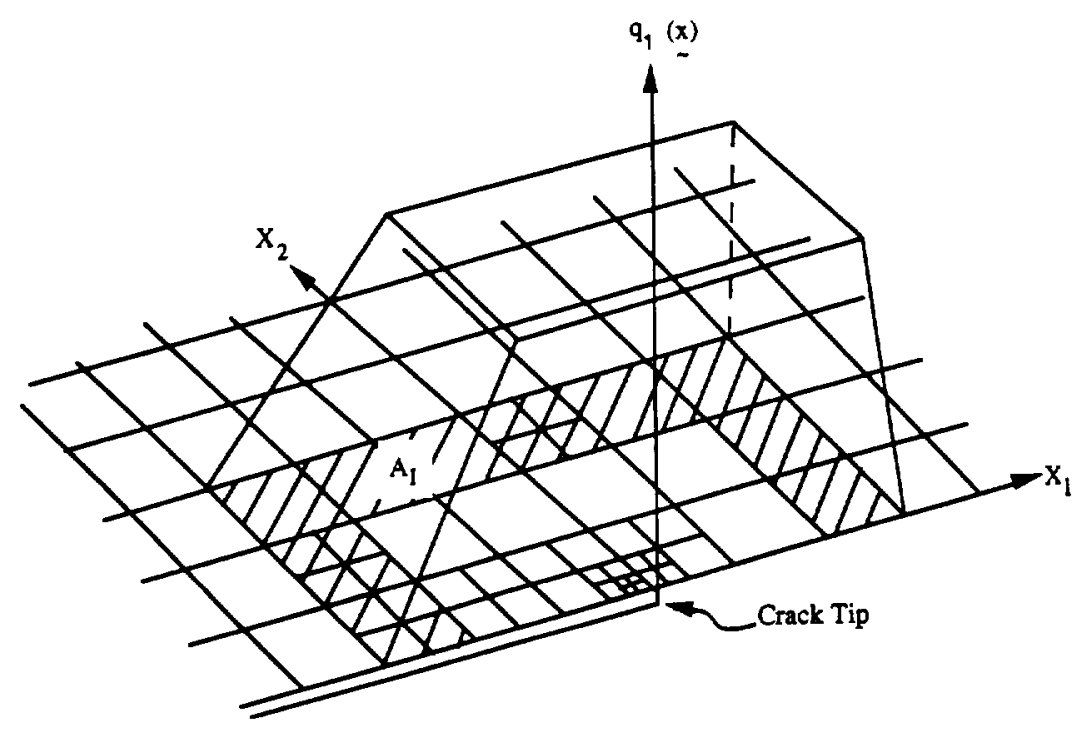

Fig. 4. Schematic definition of the $q_{1}$-function. 


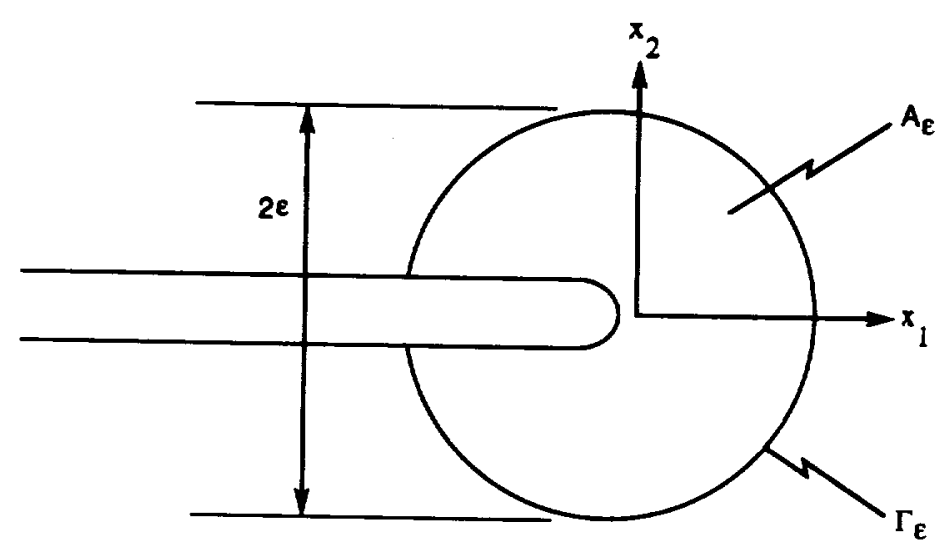

Fig. 5. Definition of the parameters involved in the definition of the components of the $J$-integral.

expression for the energy release rate; the twodimensional version of this expression reads as follows (see [26], pp. $408-409$ for the derivation):

$$
G=J=-\int_{\Lambda_{1}} P_{1 j} \frac{\partial q_{1}}{\partial x_{j}} \mathrm{~d} \Omega .
$$

Here $A_{1}$ denotes the simply connected region of integration in Fig. 6 and

$$
P_{1 j}=-\sigma_{i j} \frac{\partial u_{i}}{\partial x_{1}}-W \cdot \delta_{1 j}
$$

is the $x_{1}$-component of Eshelby's energy momentum tensor $[29,30], W$ is the strain energy density, and $q_{1}$ is a sufficiently smooth function in $A_{1}$ which is unity on the inner boundary $C_{1}$ and vanishes on the outer boundary $C_{2}$ of $A_{1}$.

A general study of crack-tip contours and domain integrals was presented by Moran and Shih $[27,28]$. In two dimensions, the energy release rate is expressed by an equivalent domain integral given by eqn (20); a schematic definition of the integration domain $A_{1}$ and the weight function $q_{1}$ is given in Fig. 4. According to [31] the basic definition of

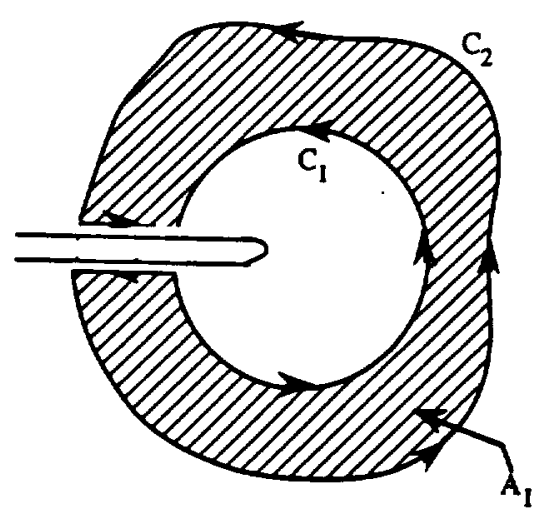

Fig. 6. Simply connected region $A_{1}$ with subsets $C_{1}$ and $C_{2}$ of the boundary.
$J$-integral components, as crack-tip parameters, is (see Fig. 5) given by

$$
J_{k}=\lim _{\epsilon \rightarrow 0} \int_{\Gamma_{\mathrm{t}}}\left[W \cdot n_{k}-\sigma_{i j} \cdot \frac{\partial u_{i}}{\partial x_{k}} \cdot n_{j}\right] \mathrm{d} \Gamma .
$$

Here $W$ is the stress-work density, $\sigma_{i j}$ are stresses, $u_{i}$ are displacements, $n_{k}$ are components of the unit normal vector at points on the contour $\Gamma$. A local crack front coordinate system is given by $x_{1}$ and $x_{2}$ : $x_{1}$ is normal to the crack front, $x_{2}$ is orthogonal to $x_{1}$. In the linear elastic case, it is possible to define SIFs through the calculation of $J_{k}(k=1,2)$. According to [32] the relationships between the $J$-integral components and the stress factors are

$$
\begin{aligned}
& K_{\mathrm{I}}=\frac{1}{2} E^{*}\left(\sqrt{J_{1}-J_{2}}+\sqrt{J_{1}+J_{2}}\right) \\
& K_{11}=\frac{1}{2} E^{*}\left(\sqrt{J_{1}-J_{2}}-\sqrt{J_{1}+J_{2}}\right),
\end{aligned}
$$

where

$$
E^{*}= \begin{cases}\frac{E}{1-v^{2}} & \text { for plane strain state } \\ E & \text { for plane stress state. }\end{cases}
$$

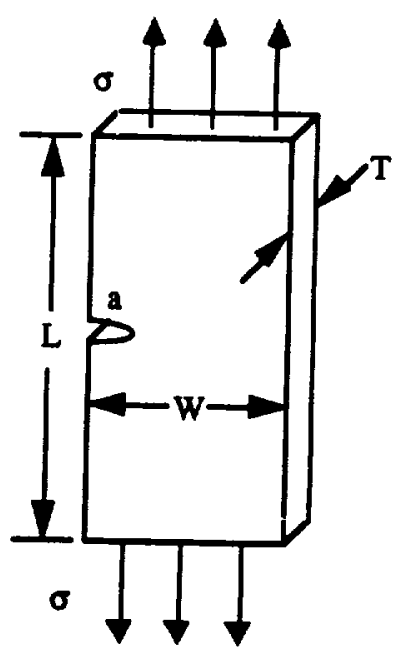

Fig. 7. Single edge crack. 


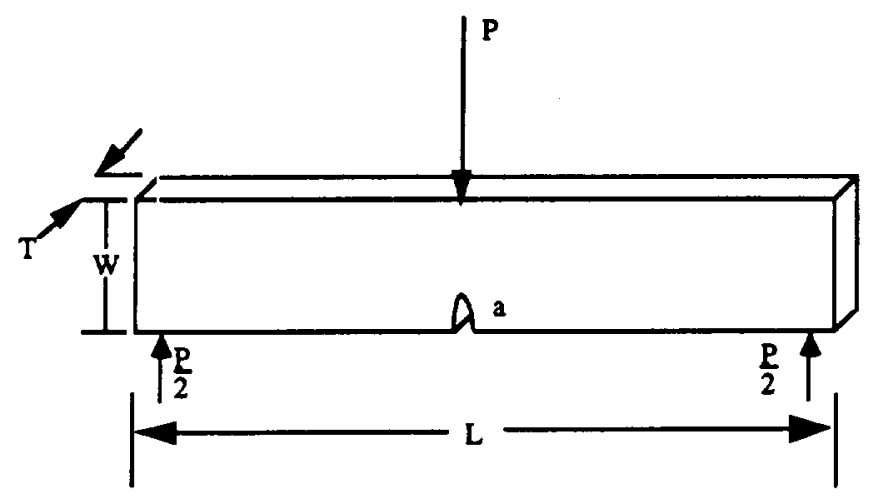

Fig. 8. Edge cracked beam.

\section{NUMERICAL EXAMPLES}

This section presents the demonstration problems. The cases consist of a single edge crack and an edge cracked beam; both cases were computed using two different crack lengths. The results for each case are shown as stress contour plots on the deformed shape scaled by a factor of 100 to clearly show crack. Fifteen to twenty levels of refinement were used to compute the SIFs using the methods shown in Sec. 3. The 'equivalent-domain integral' method was used for problems 1 and the 'discrete least-squares fits' method was used for problem 2 .

(a)

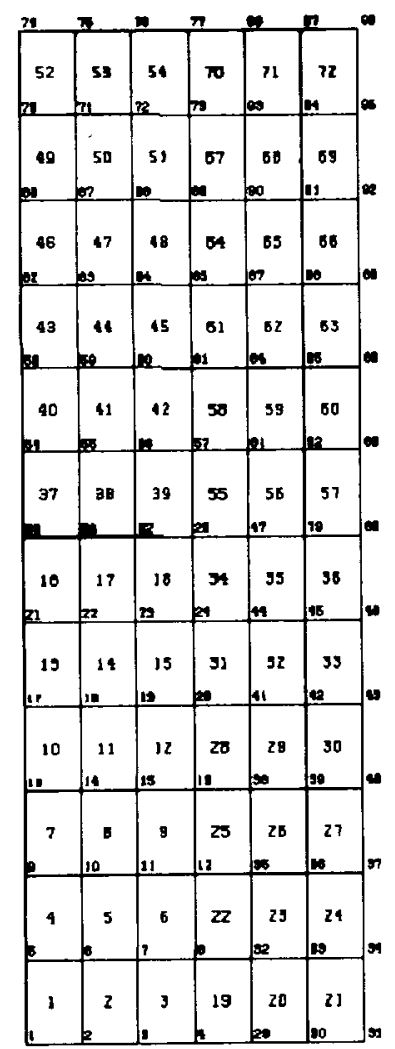

\subsection{Single edge crack (problem 1)}

Figure 7 is a schematic of this demonstration problem. The problem parameters are given as follows. Dimensions: $L=12$ inches, $W=4$ inches, and $T=1.0$ inch. Material properties: $E=10^{6} \mathrm{psi}$ and $v=0.3$. Crack lengths: $a=2.0$ and 0.2 inches. Stress distribution: $\sigma=100 \mathrm{ksi}$ (top and bottom). Mode I stress intensity factors were calculated at each crack length for the given stress distributions and both crack lengths for two cases of this problem.

4.1.1. Crack length $\mathrm{a}=2.0$ (problem $1 A)$. The representative solutions for this problem are shown in

(b)

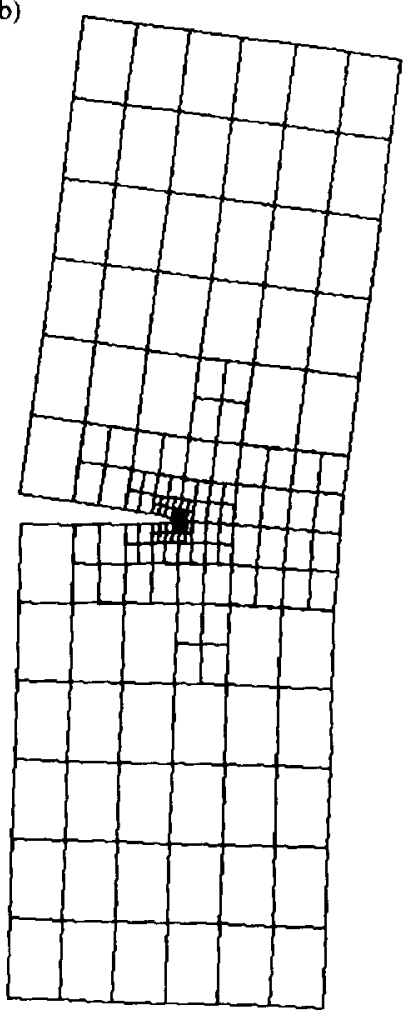

Fig. 9. Problem IA: (a) initial grid, (b) fourth level refinement grid. Note that the grids are scaled by a factor of 100 to clearly show the crack. 

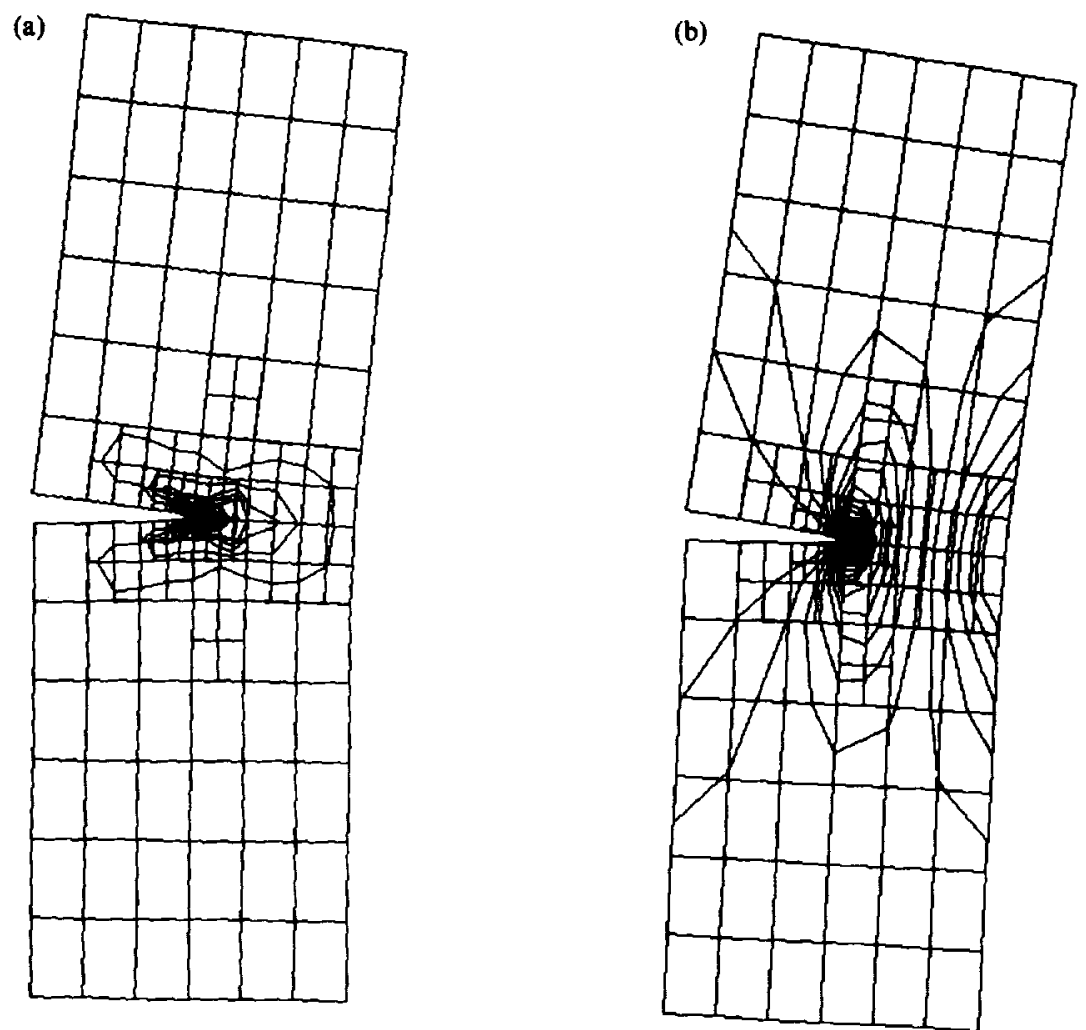

Fig. 10. Problem 1A: (a) $\sigma_{x}$ for fourth level refinement grid, (b) $\sigma_{y}$ for fourth level refinement grid. Note that the grids are scaled by a factor of 100 to clearly show the crack.

(a)

\begin{tabular}{|c|c|c|}
\hline$\pi$ & $n$ & $n$ \\
\hline 67 & 80 & 89 \\
\hline at & OS & .0 \\
\hline 51 & 62 & 63 \\
\hline 50 & 38 & 60 \\
\hline 50 & 35 & 57 \\
\hline M4 & 95 & 36 \\
\hline 91 & 32 & 33 \\
\hline 20 & 21 & so \\
\hline$\overline{z 5}$ & 26 & 27 \\
\hline 22 & 25 & 24 \\
\hline 19 & 20 & 2) \\
\hline
\end{tabular}

(b)

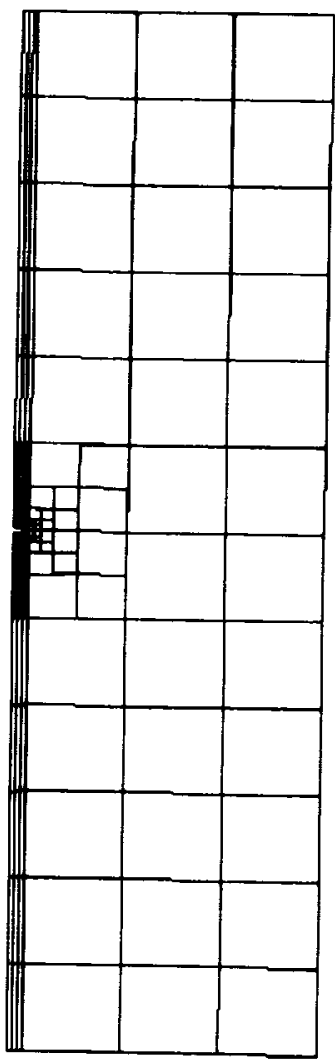

Fig. 11. Problem IB: (a) initial grid, (b) fourth level refinement grid. Note that the grids are scaled by a factor of 100 to clearly show the crack. 
Table 1. SIF comparisons for example problem 1

\begin{tabular}{cccc}
\hline & Adaptive FEM & Ref. [33] & Ref. [34] \\
\hline Problem 1A & 715 & 755 & 706 \\
Problem 1B & 87.5 & 89.9 & 89.6 \\
\hline
\end{tabular}

Figs 9 and 10. Figure 9 shows the initial grid distribution and fourth level refinement. Figure 9 shows the grid with $\sigma_{x}$ and $\sigma_{y}$ contours for the fourth level of refinement.

4.1.2. Crack length $\mathrm{a}=0.2$ (problem $1 B$ ). The representative solutions for this problem are shown in Figs 11 and 12. Figure 11 shows the initial grid distribution for this case. Figure 12 shows the grid and fourth level refinement with $\sigma_{x}$ and $\sigma_{y}$ contours for the fourth level of refinement.

4.1.3. Validation for problem 1. The 'energy release' method was used in this problem. References [33] and [34] provide formulas for estimating the SIF for this problem. The formulas used are of the form

$$
K_{1}=\sigma \cdot \sqrt{\pi \cdot a} \cdot F(h), \quad h=\frac{a}{W} .
$$

The references use fourth-order expansion in $h$ to determine the multiplier $F$. Table 1 compares $K_{1}$ obtained from the adaptive finite element code and the analytical formulas from the references.

(a)

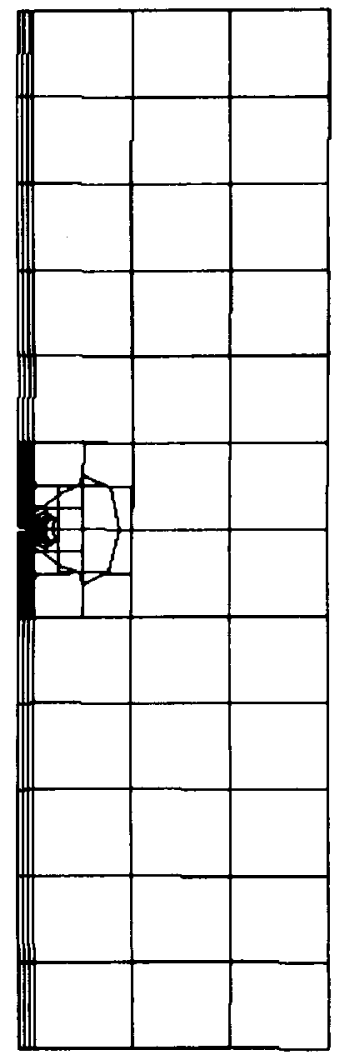

\subsection{Edge cracked beam (problem 2)}

Figure 8 is a schematic of this problem. The problem parameters are given as follows. Dimensions: $W=2$ inches, $L=4 W=8$ inches, $T=$ 1.0 inch. Material properties: $E=10^{6} \mathrm{psi}, v=0.3$. Crack lengths: $a=0.5$ and 0.1 inches. Stress distribution: $P=5000 \mathrm{lb}$ (point load). Mode I stress intensity factors were calculated for both crack lengths for two cases of this problem.

4.2.1. Crack length $\mathrm{a}=0.5$ (problem $2 A$ ). Figure 13 shows the initial and fourth level refinement grid distribution. The solutions of fourth level of refinement for this problem are shown in Fig. 14 to show the grid with $\sigma_{x}$ and $\sigma_{y}$ contours for this level of refinement.

4.2.2. Crack length $\mathrm{a}=0.1$ (problem $2 B$ ). The solutions of this problem are shown in Figs 15 and 16 . Figure 16 shows the grid with $\sigma_{x}$ and $\sigma_{y}$ contours for the fourth level of refinement.

4.2.3. Validation for problem 2. The 'least squares' method was used in this problem. References [33] and [34] provide formulas for estimating the SIF for this problem. The formula used are of the form

$$
\begin{aligned}
& K_{\mathrm{L}}=\sigma \cdot \sqrt{\pi \cdot a} \cdot F(h), \quad h=\frac{a}{W} \\
& \sigma=\frac{6 \cdot M}{W^{2} T}, \quad M=\frac{P L}{4} .
\end{aligned}
$$

(b)

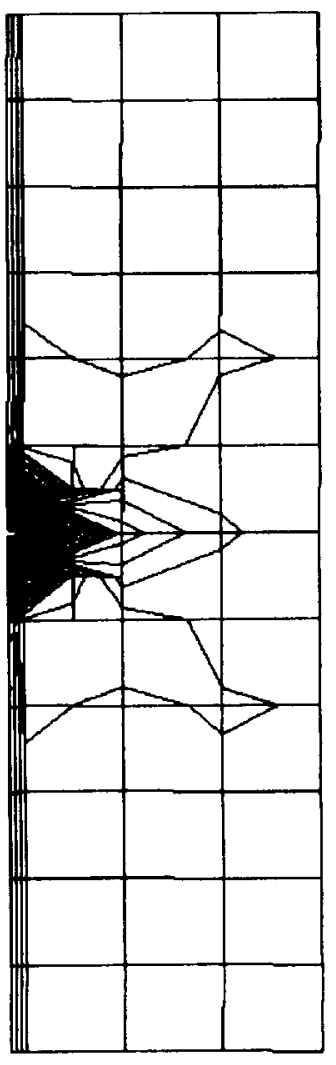

Fig. 12. Problem IB: (a) $\sigma_{x}$ for fourth level refinement grid, (b) $\sigma_{y}$ for fourth level refinement grid. Note that the grids are scaled by a factor of 100 to clearly show the crack. 
(a)

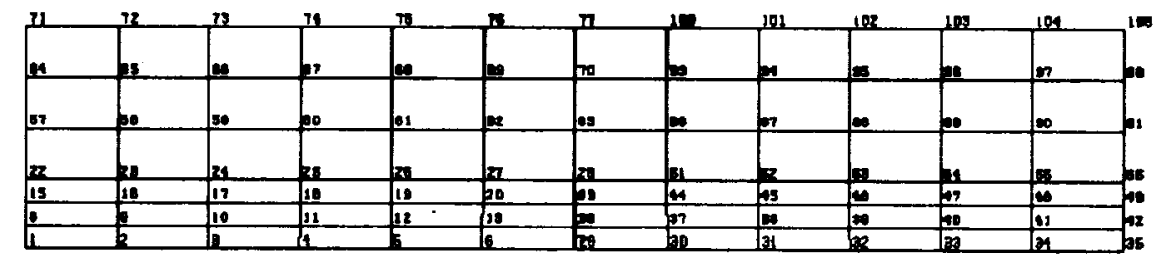

(b)

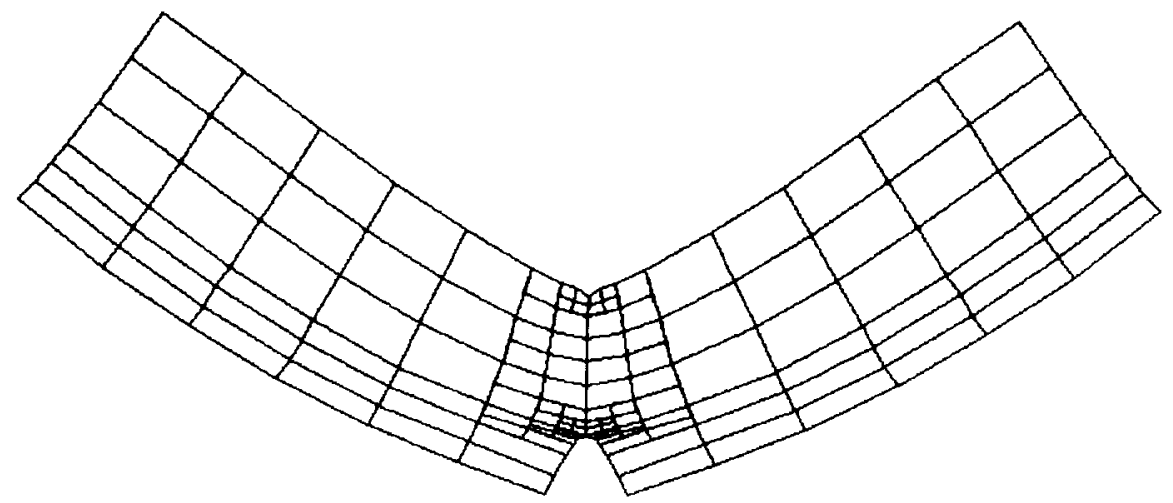

Fig. 13. (a) Initial grid; (b) fourth level refinement grid of problem $2 \mathrm{~A}$.

(a)

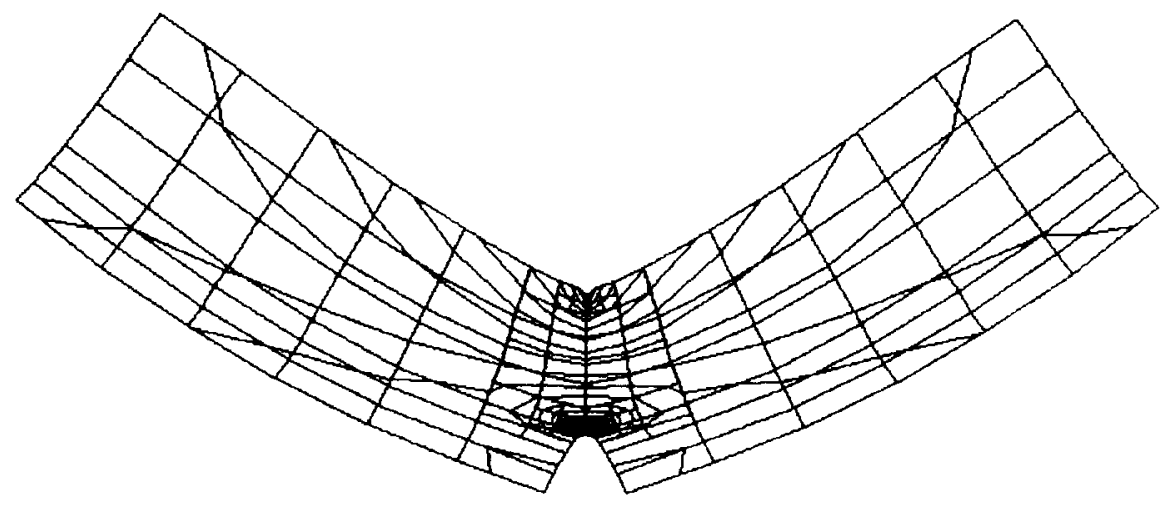

(b)

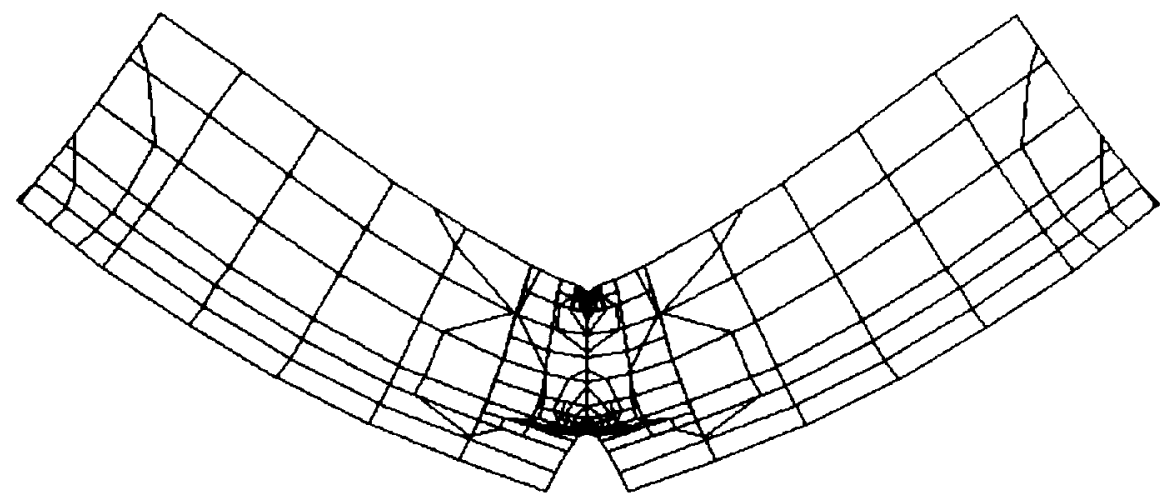

Fig. 14. (a) $\sigma_{x}$ on the fourth level refinement grid; (b) $\sigma_{y}$ on the fourth level refinement grid of problem 2A. (Note: grid scaled by a factor of 100 to clearly show the crack.) 
Solution-adaptive finite element methods

443

(a)

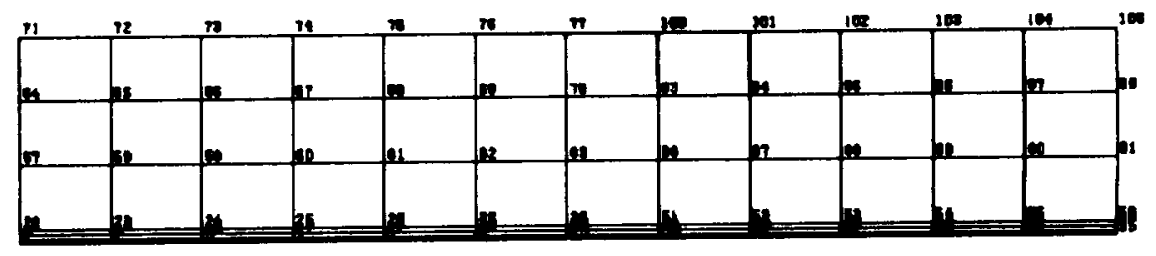

(b)

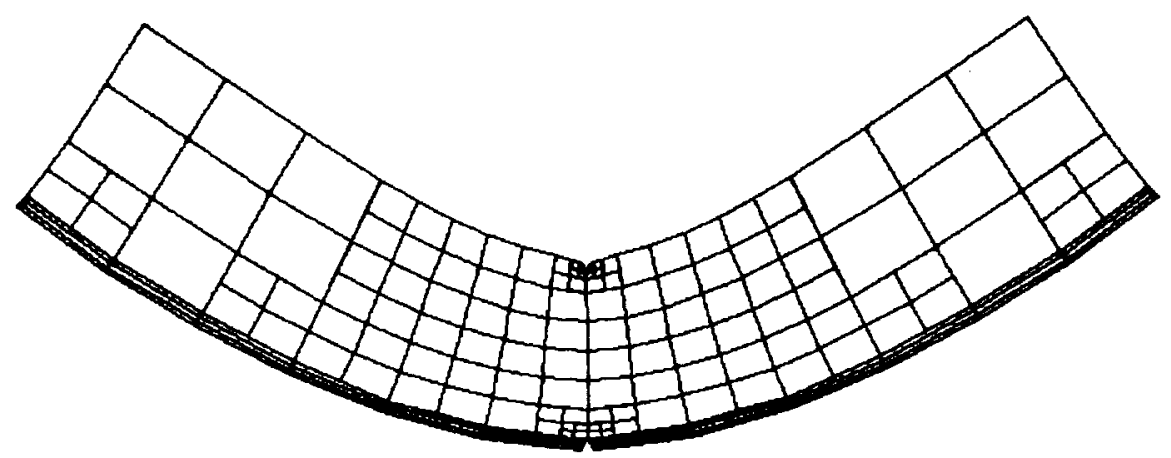

Fig. 15. (a) Initial grid; (b) fourth level refinement grid of problem 2B.

(a)

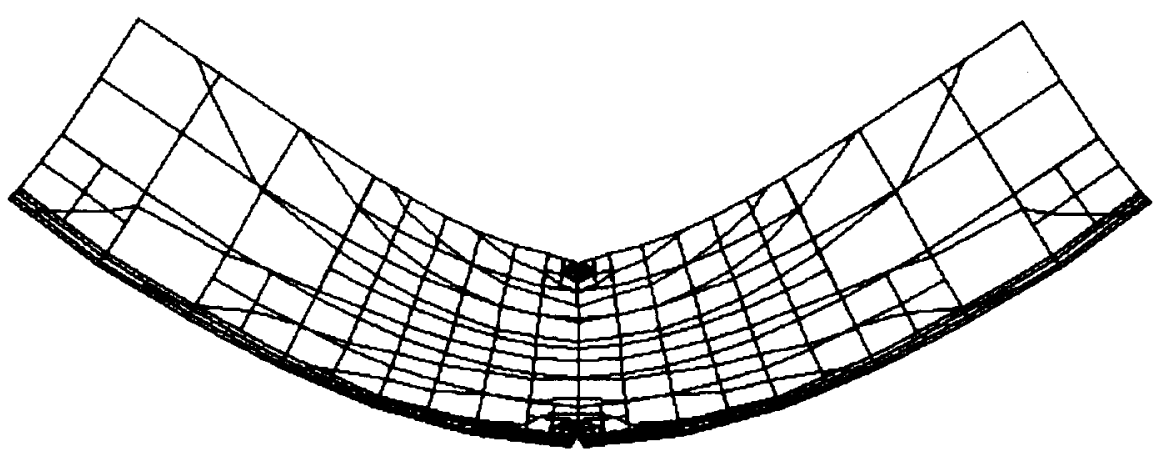

(b)

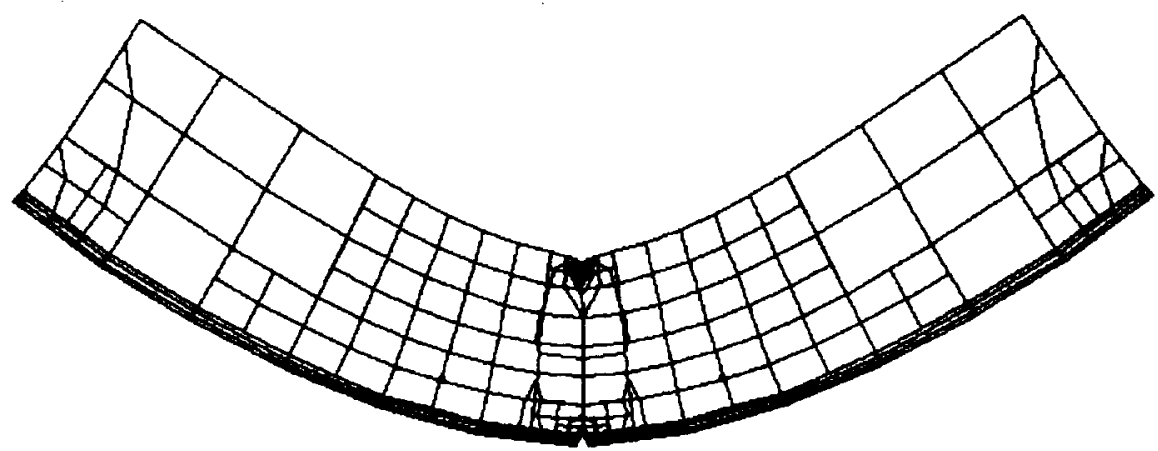

Fig. 16. (a) $\sigma_{x}$ on the fourth level refinement grid; (b) $\sigma_{y}$ on fourth level refinement grid of problem 2B. 
Table 2. SIF comparisons for example problem 2

\begin{tabular}{lccc}
\hline & Adapative FEM & Ref. [33] & Ref. [34] \\
\hline Problem 2A & 17,901 & 18,917 & 20,301 \\
Problem 2B & 8611 & 8418 & 8985 \\
\hline
\end{tabular}

The references uses the fourth-order expansions in $h$ to determine the multiplier $F$. Table 2 compares $K_{\mathrm{I}}$ obtained from this study with $K_{1}$ obtained using the analytical formula from the references.

\section{CONCLUSIONS}

A solution-adaptive computational method has been developed and implemented for accurately determining the SIFs of two-dimensional crack configurations with predetermined crack locations and loading conditions. This new method and procedures have been validated for demonstration problems showing reasonably good agreement with analytical formula and can be used for performing the two-dimensional fracture mechanics analysis in complex structures. Also, this study demonstrated the feasibility of the methodology developed to be extended in the three-dimensional analyses.

Acknowledgements - The second and third authors were supported by NAS8-38907. The authors wish to thank J. M. Price and C. D. Wilson of NASA/MSFC for helpful discussions on selecting the example problems.

\section{REFERENCES}

1. E. R. A. Oliveira, Optimization of finite element solutions. Proceedings of the Third Conference on Matrix Methods in Structural Mechanics, Wright-Patterson AFB, Ohio (1971).

2. L. Demkowicz, Some remarks on moving finite element methods. Comput. Meth. Appl. Mech. Engng 46, 339-349 (1984).

3. B. M. Herbst, A. R. Mitchell and S. W. Schoombie, A moving Petrov-Galerkin method for transport equations. Int. J. Numer. Meth. Engng 18, 1321-1336 (1982).

4. J. S. Kaminski, P. A. Clitherow and G. J. Stuk, Integrating expert systems with conventional applications. Computers in Engineering 1, 225-229 (1988).

5. K. Miller, Recent results on finite element methods with moving nodes. ARFEC, Lisbon (1984).

6. K. Miller and R. N. Miller, Moving finite elements I. SIAM J. Numer. Anal. 18, No. 6 (1981).

7. A. C. Mueller and G. F. Carey, Continuously deforming finite element methods. Int. J. Numer. Meth. Engng 21, 2099-2130 (1985).

8. A. J. Wathen, M. J. Baine and K. W. Morton, Moving finite element methods for the solution of evolutionary equations in one and two space dimensions. MAFELAP (1984).

9. L. Demkowicz, J. T. Oden and P. Devloo, An $h$-type mesh refinement strategy based on a minimization of interpolation error. Comput. Meth. Appl. Mech. Engng $53,67-69$ (1985)

10. J. T. Oden and L. Demkowicz, Adaptive finite element methods for complex problems in solid and fluid mechanics. Computational Mechanics, ITT, Bombay (1985).

11. J. T. Oden, T. Strouboulis and P. Devloo. In Adaptive Finite Element Methods for Compressible Flow (Edited by T. E. Tezduyar). AMD Monograph (1986).
12. J. T. Oden, L. Demkowicz, T. Strouboulis and P. Devloo, Adaptive methods for problems in solid and fluid mechanics. In Accuracy Estimates and Adaptive Refinements in Refinement Element Computations (Edited by I. Babuska et al.). John Wiley, Chichester (1986).

13. J. M. Bass and J. T. Oden, Adaptive finite element methods for a class of evolution problems in viscoplas. ticity. Int. J. Engng Sci. 25, 623-653 (1987)

14. A. W. Craig, J. A. Zhu and O. C. Zienkiewicz, $A$ posteriori error estimation methods using hierarchical finite element bases. Notes CR/483/84 of the Institute for Numerical Methods in Engineering, University College of Swansea, Swansea, U.K. (1994).

15. B. A. Szabo, Mesh design for the $p$-version of the finite element methods. Comput. Method. Appl. Mech. Engng 55, No. $1 / 2$ (1986).

16. B. A. Szabo, P. K. Vasu and M. P. Rossow, Adaptive finite element analysis based on $p$-convergence. NASA Conference Publications 2059, pp. 43-50 (1978).

17. B. A. Szabo and I. Babuska, Stress approximations by the $h$ - and $p$-versions of the finite element method. Report WU/CCM-82/1, Center for Computational Mechanics, Washington University (1982).

18. O. C. Zienkiewicz and A. W. Craig, Adaptive mesh refinement and a posteriori error estimation for the $p$-version of the finite element method. In Adaptive Computational Methods for Partial Differential Equations (Edited by I. Babuska et al.). SIAM Publications, Philadelphia, PA (1983).

19. I. Babuska, W. Gui and B. Szabo, Performance of the $h, p$, and $h-p$ versions of the finite element method. Preprint (1984).

20. L. Demkowicz, J. T. Oden, W. Rachowicz and O. Hardy, Toward a universal $h-p$ adaptive finite element solver. TICOM Report, The University of Texas at Austin (1989)

21. L. Demkowicz, J. T. Oden, W. Rachowicz and O. Hardy, Toward a universal $h-p$ adaptive finite element strategy, part I: constrained approximation and data structure. Comput. Meth. Appl. Mech. Engng 77, 79-112 (1989).

22. J. T. Oden, L. Demkowicz, W. Rachowicz and T. A. Westermann, Toward a universal $h-p$ adaptive finite element strategy, part II: a posteriori error estimation. Comput. Meth. Appl. Mech. Engng 77, 113-180 (1989)

23. W. Rachowicz, J. T. Oden and L. Demkowicz, Toward a universal $h-p$ adaptive finite element strategy, part III: design of $h-p$ meshes. Comput. Meth. Appl. Mech. Engng 77, 181-212 (1989).

24. P. G. Ciarlet and P. A. Raviart, Interpolation theory over curved elements with applications to finite methods. Comput. Meth. Appl. Mech. Engng 1, 217-249 (1972).

25. J. T. Oden and G. F. Carey, Finite Elements: Mathematical Aspects, Vol. IV. Prentice-Hall (1983).

26. F. Z. Li, C. F. Shih and A. Needleman, A comparison of methods for calculating energy release rates. Engng Fract. Mech. 21, 405-421 (1985)

27. B. Moran and C. F. Shih, A general treatment of crack tip contour integrals. Int. J. Fract. 35, 295-310 (1987).

28. B. Moran and C. F. Shih, Crack tip and associated domain integrals from momentum and energy balance. Engng Fract. Mech. 27, 615-642 (1987).

29. J. D. Eshelby, The continuum theory of lattice defects. In Solid State Physics (Edited by F. Seitz and D. Turnbull), Vol. III, pp. 79-144. Academic Press, New York (1956).

30. J. D. Eshelby, The energy momentum tensor in continuum mechanics. In Inelastic Behavior of Solids (Edited by M. F. Kanninen et al.), pp. 77-114. McGraw-Hill, New York (1970). 

31. G. P. Cherepanov, Mechanics of Brittle Fracture. 33. D. Broek, Elementary Engineering Fracture Mechanics. McGraw-Hill, New York (1979).

Sijthoff \& Noordhoff (1982) $\begin{array}{ll}\text { 32. G. P. Nikishkov and V. A. Vainshtok, Method of virtual } & \text { 34. H. Tada, P. C. Paris and G. R. Irwin, The Stress } \\ \text { crack growth for determining stress intensity factors } K_{1} & \text { Analysis of Cracks Handbook. Del Research Corpor- }\end{array}$ and $K_{11}$. Strength of Materials 12, 696-701 (1980). ation, Hellertown, PA (1973). 